\title{
Improving the Recording of Diagnoses in Primary Care with Team Incentives: A Controlled Longitudinal Follow-Up Study
}

\author{
Tuomo Lehtovuori, ${ }^{1}$ Timo Kauppila ${ }^{(D)},{ }^{2,3}$ Jouko Kallio, ${ }^{1}$ Anna M. Heikkinen, ${ }^{4}$ \\ Marko Raina, ${ }^{3}$ Lasse Suominen, ${ }^{1}$ and Reijo Sund ${ }^{5}$ \\ ${ }^{1}$ City of Espoo, Administration of Primary Care, Espoo, Finland \\ ${ }^{2}$ Department of General Practice and Primary Healthcare, University of Helsinki, P.O. Box 20, \\ Tukholmankatu 8 B, 00014 Helsinki, Finland \\ ${ }^{3}$ City of Vantaa, Administration of Primary Care, Vantaa, Finland \\ ${ }^{4}$ Department of Oral and Maxillofacial Diseases, Institute of Dentistry, University of Helsinki, P.O. Box 41, 00014 Helsinki, Finland \\ ${ }^{5}$ Centre for Research Methods, Department of Social Research, University of Helsinki, P.O. Box 18, \\ Unioninkatu 35, 00014 Helsinki, Finland
}

Correspondence should be addressed to Timo Kauppila; timo.kauppila@fimnet.fi

Received 23 June 2017; Revised 23 October 2017; Accepted 9 January 2018; Published 20 February 2018

Academic Editor: Nikolaos Siafakas

Copyright (C) 2018 Tuomo Lehtovuori et al. This is an open access article distributed under the Creative Commons Attribution License, which permits unrestricted use, distribution, and reproduction in any medium, provided the original work is properly cited.

Introduction. We studied whether primary care teams respond to financial group bonuses by improving the recording of diagnoses, whether this intervention leads to diagnoses reflecting the anticipated distribution of diseases, and how the recording of a significant chronic disease, diabetes, alters after the application of these bonuses. Methods. We performed an observational register-based retrospective quasi-experimental follow-up study with before-and-after setting and two control groups in primary healthcare of a Finnish town. We studied the rate of recorded diagnoses in visits to general practitioners with interrupted time series analysis. The distribution of these diagnoses was also recorded. Results. After group bonuses, the rate of recording diagnoses increased by 17.9\% (95\% CI: 13.6-22.3) but not in either of the controls ( -2.0 to $-0.3 \%)$. The increase in the rate of recorded diagnoses in the care teams varied between $14.9 \%$ (4.7-25.2) and 33.7\% (26.6-41.3). The distribution of recorded diagnoses resembled the respective distribution of diagnoses in the former studies of diagnoses made in primary care. The rate of recorded diagnoses of diabetes did not increase just after the intervention. Conclusions. In primary care, the completeness of diagnosis recording can be, to varying degrees, influenced by group bonuses without guarantee that recording of clinically significant chronic diseases is improved.

\section{Introduction}

Financial incentives can be defined as "all the rewards agents (physicians or the physician organization) receive conditional on their measured (explicitly or implicitly) performance or behavior" [1]. The system by which physicians are paid may affect their professional practice and decision-making $[2,3]$. Therefore, differently tailored payment systems for general practitioners (GPs) have been used to achieve the desired policy objectives, such as improving the quality of care or recruitment to underserved areas $[2,3]$. There is ample recent research about how pay-for-performance systems to GPs alter their clinical activities [4-10]. They can also be used to reduce inequalities in the delivery of clinical care related to area deprivation [4]. There are quite autonomous actors other than physicians, such as nurses who specialize in the treatment of diabetes [11-14], who may influence the clinical practice of their organization dramatically and even improve the quality of care [11, 14]. Therefore, disciplines other than GPs might well be considered as targets for financial incentives.

Insufficient recording of diagnoses may hamper planning of healthcare and adequate allocation and management of resources [15] and improving the recording of chronic diseases might theoretically serve as one of the first targets in improving the quality of care $[15,16]$. Therefore, the administration of the primary healthcare of Espoo City considered 
the recording of diagnoses in only $40-60 \%$ of doctor visits to be insufficient [17]. Former studies suggested that financial incentives to GPs increased the recording of diagnoses [5, 7] and a preliminary analysis suggested that group bonuses could do the same [17]. With financial group bonuses to care teams, the administration of Espoo primary care wanted to improve the recording of diagnoses, especially the recording of diabetes diagnoses. Diabetes is known to require considerable care and causes a lot of costs [16, 18-20].

The main aim of this present study was to quantify the extent of the effect of group bonuses on improvement in the level of marking diagnoses in the patient chart system. We examined whether all teams responded equally to this intervention. The administration was also concerned about the adequacy of the marked diagnoses because any diagnosis recording, even inadequate, produced financial rewards. Therefore, we investigated the range of diagnoses which were recorded to find out whether the present financial intervention produced data which reflected the distribution of diagnoses in real clinical life in primary care and thus provided valid data about public health. As an example of a significant chronic public disease, the recording of diabetes [16, 18-20] was monitored to find out whether this disease was recorded more frequently after the present intervention. To provide impression about costs group bonus incurred for Espoo primary care, the percentage of staff receiving bonuses was recorded, as well as the mean bonus per year for one staff member.

\section{Methods}

2.1. Design and Setting. The present research is a retrospective longitudinal quasi-experimental study with a before-andafter design in 5 primary healthcare areas, with 3-6 care teams (cells) each, in Espoo (230,000 and 254,000 inhabitants in 2006 and 2012, resp.). Altogether, the number of cells was 23. There were 6-8 doctors and 6-8 nurses in each cell. The precise number of doctors and nurses varied slightly over the study period. More detailed information about the functions and frequency of use in Espoo primary care at the time of this study was described previously [17].

No ethical approval was required because this study was made directly on a computer from the patient register without identifying the patients (https://rekisteritutkimus.wordpress .com/luvat-ja-tietosuoja/). The register keeper (the health authorities of Espoo and Vantaa, 23.8.2016) granted permission to carry out the study. The report generator automatically allowed following the monthly number of recorded diagnoses for each individual doctor and therefore also by each individual cell.

As control data, we had the respective data on the recording of monthly diagnoses from two different primary care units where there was a similar cell structure but no team incentives were applied. Dental primary care of Espoo was chosen because both somatic and dental primary care are under the same administration, and we wanted to see whether desired practices disperse to other parts of the same primary care system without actual intervention. The primary care of Vantaa city was chosen because it resembles Espoo in its location and number of inhabitants (about 200,000 inhabitants, located neighboring Helsinki, the capital of Finland).
2.2. Intervention. The chosen intervention to increase the number of recorded diagnoses was to pay bonuses to all members of the care teams who met their target. The administration of Espoo primary care defined the focus areas and their goal levels at the start of 2005 and improvement in recorded diagnoses on the patient charts was chosen as the main goal. Before 1.3.2005, no group bonuses to the cells were delivered. To commit the staff to the change in function, a multidisciplinary team contract was signed with the members of the cells. The contract defined the rules and approaches to the functions of the cells. The team contracts were signed by all of the five service areas for the period 1.3.2005-30.5.2005, which was considered to be the time of the start of the intervention. After signing this contract, the cells were able to aim for group bonuses. This meant that, to get a group bonus, a care team had to take care that diagnoses were recorded in more than $75 \%$ of all doctor visits of that team.

2.3. Primary and Secondary Outcomes. The proportion of monthly doctor visits having recorded diagnoses was selected as the main measure to study the effect of implementing group bonuses. Diagnoses were recorded with the ICD-10 system by the doctors.

To study the effects of group bonuses at the individual cell level, the team-based monthly percentage of visits having marked diagnoses was derived from electrical patient charts from each care team in Espoo primary care. We calculated the monthly variation of these percentages in each cell during the year 2006 when the intervention was already fully functioning. The variation between care teams was analyzed using these aggregated cell-level percentages. To establish whether the team incentive system had altered the recording of diagnoses of chronic diseases, the monthly numbers of diabetes diagnoses (ICD-10 codes E10 and E11) were also studied in Espoo health center. Simultaneously, all diagnoses recorded during 2006 were also gathered.

The costs of group bonuses were obtained from years 2005 and 2006 from the payroll system of the social and health bureau of Espoo. The percentage of staff receiving bonuses and annual bonus per staff member were recorded.

2.4. Data Extraction. The effect of the intervention on the proportion of monthly doctor visits with recorded diagnoses was continuously monitored for a two-year time period before intervention and 1.5 years after it. The data about the recorded diagnoses was specifically derived from the electronic Effica patient chart system (Tieto Ltd., Helsinki, Finland) from which the data were reliably obtainable from 1.5.2003. The control data from Espoo dental care were similarly obtainable from 1.5.2003. The control data from Vantaa health center were obtained from the graphic Finstar patient chart system (Logica Ltd., Helsinki, Finland). The Effica and Finstar patient chart systems have a similar setting in the site where the diagnosis is supposed to be marked. Writing the three first symbols (letters when using directly a diagnosis or a letter and two numbers when using directly an ICD-10 code) opens automatically a list of all putative diagnoses with that symbol combination. Then, the GP or dentist can choose by double-clicking the desired diagnosis from these options. 


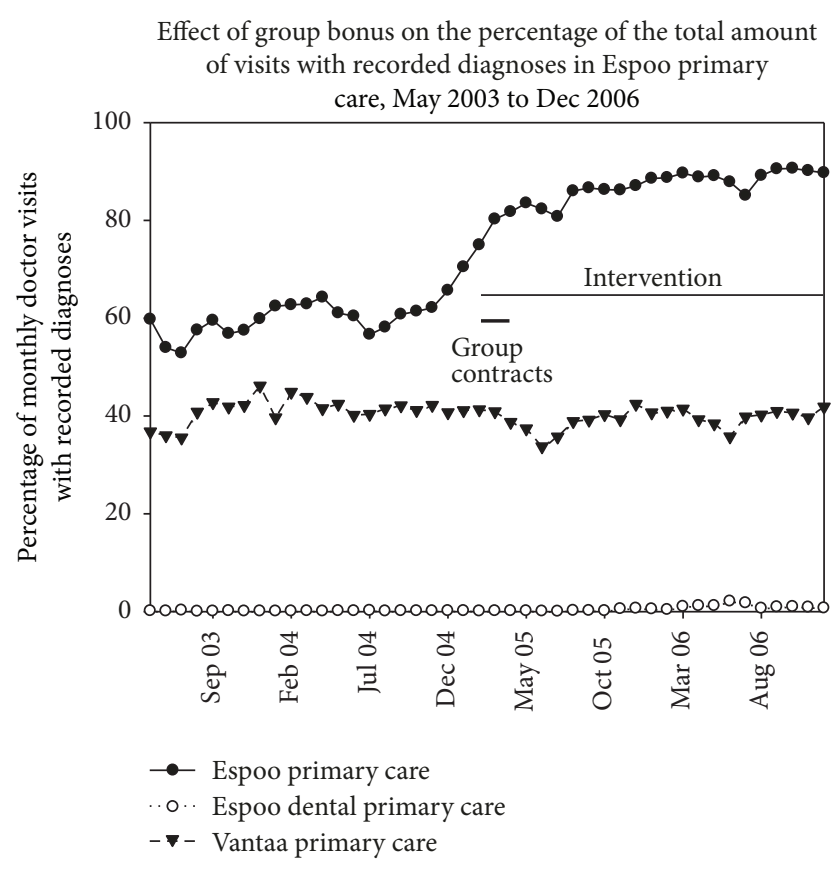

FIGURE 1: Effect of group bonus on the percentage of total amounts of visits to doctors with recorded diagnoses in Espoo primary care and controls. Follow-up time is May 2003 to Dec 2006.

2.5. Statistical Analysis. To study the effect of intervention on the frequency of marking diagnoses in GP visits, we used interrupted time series (ITS) ARIMA model [21] to compare the percentages of monthly doctor visits with recorded diagnoses before and after intervention. The same testing methods were used when these proportions were compared with the control units (Espoo dental healthcare and Vantaa primary care).

The variations between care teams in Espoo primary care were analyzed using the aggregated cell-level percentages in 2006 and performing One-Way Repeated Measures- (RM-) ANOVA on ranks with suitable corrections (Tukey) for multiple comparisons or when following the development of an individual care team as a function of time.

To study whether the team incentive system altered the recording of diagnoses of chronic diseases, the monthly number of diabetes diagnoses (ICD-10 codes E10.** and E11.**) was also analyzed with One-Way Repeated Measures Analysis of Variance followed by Bonferroni correction for multiple comparisons.

\section{Results}

Based on the ITS analyses, the proportion of recorded diagnoses in Espoo primary care increased on average by $17.9 \%$ units (95\% CI: 13.6-22.3; $P<0.001$, ITS analysis) from $59-70 \%$ up to $90 \%$ after applying group bonuses, while there was no increase in the controls (Vantaa primary care $-2.0 \%$ [-4.1-0.1] and Espoo dental care $-0.3 \%$ [-1-0.4]; see Figure 1).

During intervention in 2006, there was still considerable variation (Figure 2(a)) between different cells of Espoo health center in the recording of diagnoses during monthly doctor visits $(P<0.001$, Figure $2(\mathrm{a}))$. Despite this difference in the level of recorded diagnoses, all the cells improved their performance after intervention, and in the most active cell, Cell 1, this improvement was $33.7 \%$ (26.6-41.3; $P<0.001$, ITS analysis), while in the least active unit, Cell 11, it was less than half of that (14.9\% [4.7-25.2; $P=0.007]$, Figure 2(b)).

Altogether, 2,984 different ICD-10 diagnoses were assessed during the year 2006 by Espoo GPs. The total number of assessed diagnoses was 73,912. The distribution of the most used diagnoses in 2006 is described in Table 1. Most of the visits concerned mild respiratory infections, elevated blood pressure, low back pain, type II diabetes, and infectious gastroenteritis. The median rate of monthly doctor visits, in which diabetes diagnoses were recorded, doubled after intervention $(P<0.001$, RM-ANOVA, median: 208; IQR: $96.8)$ in 2006 when compared with preceding years (2003 [108; 71.5], 2004 [117; 29.5], or 2005 [134; 35]). According to ITS analysis, there was, however, no statistically significant intervention effect (1\% [95\% CI: -2.3-4.2]; Figure 3). During the same follow-up period, the number of all monthly doctor visits varied between 21,506 (95\% CI: 20,072-22,941 in 2004) and $22,243(20,657-23,827$ in 2005$)$ visits $(P=0.24$, RMANOVA).

The annual bonus per staff member proved to be about 700 euros (Table 2). The percentage of staff reaching the group bonus was about $50 \%$ of the total staff.

\section{Discussion}

Multidisciplinary rewarding with financial incentives, for example, group bonus with team contracts, improved the rate of marking of diagnoses in the patient charts by about $18 \%$. This improvement was not, however, observed with a chronic disease diagnosis, diabetes. There were still considerable variations between the cells in the levels of recording the diagnoses after applying group bonuses. All obtained diagnoses accurately reflected the anticipated distribution of diseases in Finnish primary care.

In accordance with the present findings regarding group bonuses, former studies suggested that financial incentives to GPs increased the recording of diagnoses and thereby the recording of diseases [5, 7]. Various and numerous administrational factors and management problems [17] were supposed to create hindrances to the proper recording of patient data, but group bonuses seemed to overcome their effects. The present data and some recent studies $[6,22,23]$ suggest that financial incentives may be used to alter the behavior of GPs towards improving the quality of care. At least some aspects of quality, for example, indirect indicators of the quality of care, may show an improvement with pay-for-performance systems while patient outcomes may not necessarily do so $[6,10]$. Taken together with the former results, our data support the view that financial incentives clearly modify system centered indicators of quality of care [24-26].

The present intervention was aimed at ensuring that the diagnoses were marked for projects directed towards improving the management of chronic illnesses in Espoo primary care [17]. Group bonuses were not, however, necessarily the cause of the observed increase in the recording 

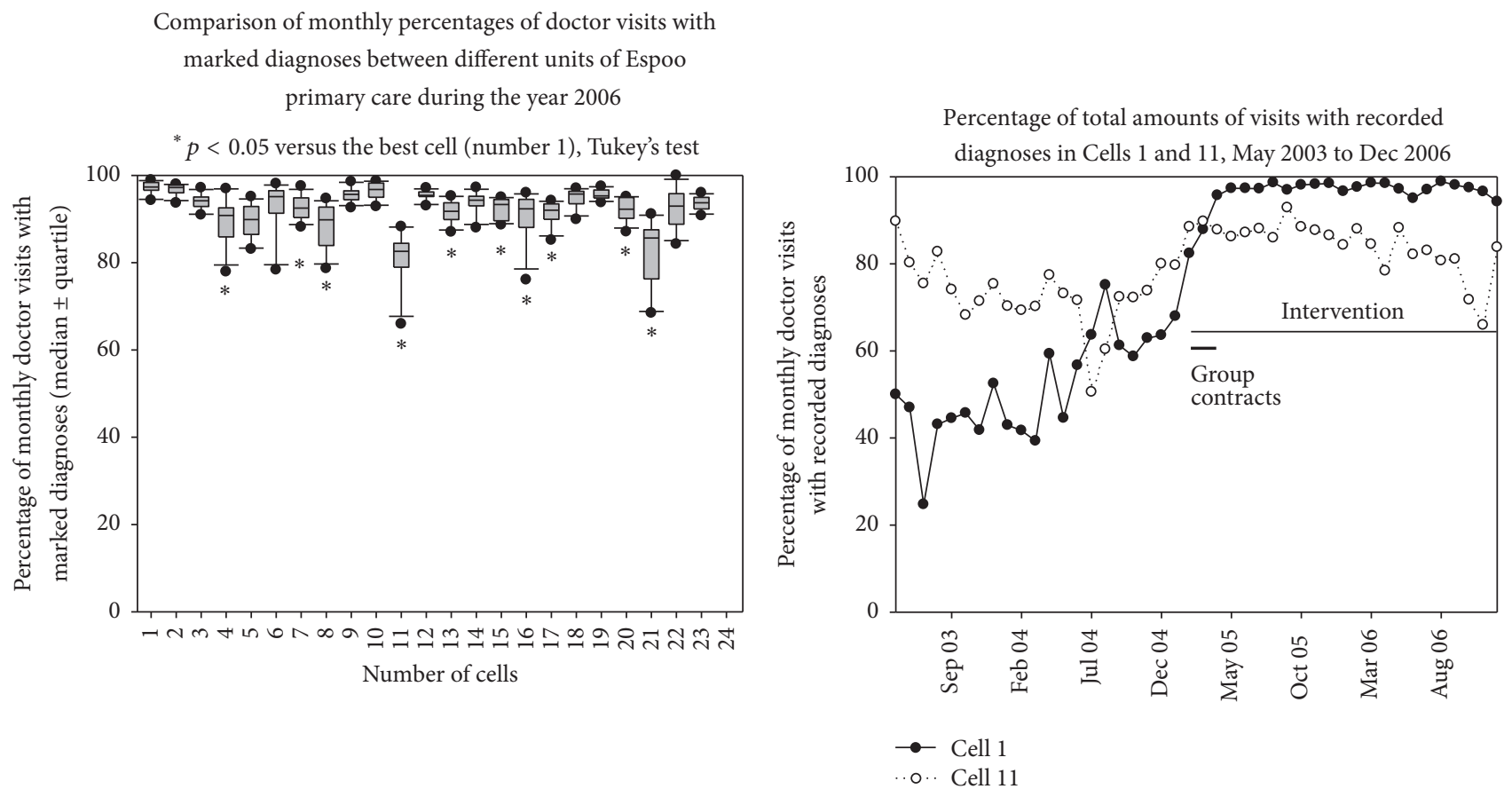

(a)

(b)

Figure 2: (a) Comparison of monthly percentages of visits to GPs with recorded diagnoses between different units of Espoo primary care during the year 2006. Median and $25 \%$ and $75 \%$ quartiles are presented with a box plot, 10 and $90 \%$ limits are presented with brackets, and the lowest and highest values are presented with dots. (b) Effect of group bonus on the percentage of total amounts of visits to GPs with recorded diagnoses in Cells 1 and 11. Follow-up time is May 2003 to Dec 2006.

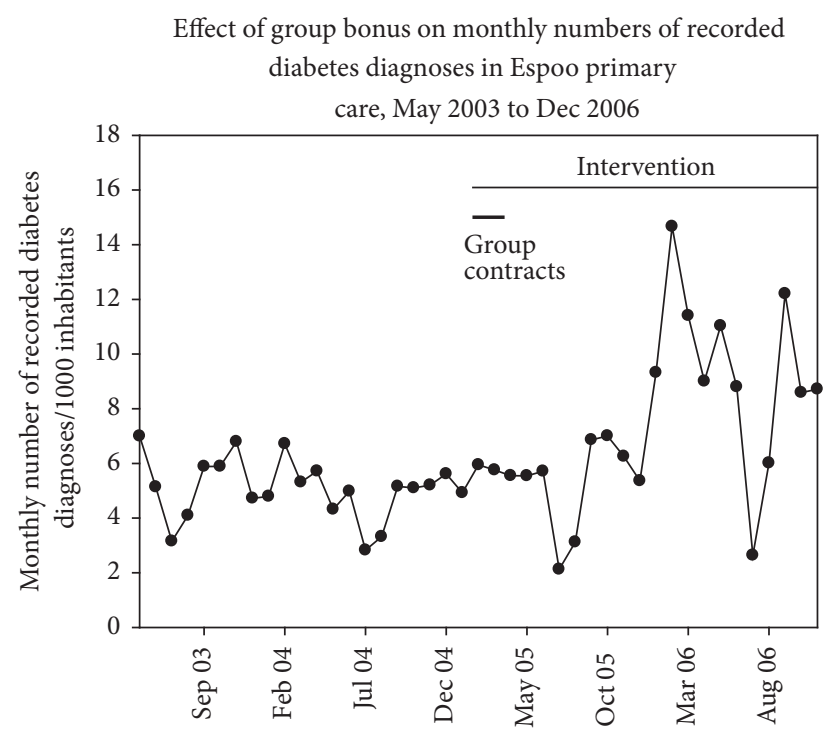

FIGURE 3: Effect of group bonus on monthly numbers of recorded diabetes diagnoses in visits to GPs. Follow-up time is May 2003 to Dec 2006.

of an important public health problem, namely, diabetes. An alternative explanation is that group bonuses were effective in increasing the recording of diabetes but that these diagnoses occurred so seldom and the change was so slow that ITS analysis failed to detect it. Nevertheless, it is not confirmed whether group bonuses are effective primers in primary care interventions which are directed towards chronic public diseases.

We cannot answer the question of why group bonuses were more effective in enhancing the recording of diagnoses in some teams and less effective in others with the present data. Roughly 60 euros extra per month is a considerable addition to salary for other members of the team than GPs. To offset "free riders" and to avoid dilution of the incentive, the teams must be relatively small [27], which fitted well with the present care teams of 16 persons or less. This relatively small size of the care teams (e.g., cells) could have created group pressure motivating the whole team to improve performance. Money is an essential factor, but other mechanisms, such as group pressure, may play a role in financial incentive-based interventions. To support this, partial withdrawal of financial incentives did not lead to a deterioration of results obtained with a GP-based pay-for-performance intervention [9] and the mean team-based rate of recording diagnoses decreased only a little after total withdrawal of group bonuses [17].

In the present study, most of the visits (Table 1) concerned mild respiratory infections, elevated blood pressure, low back pain, and type II diabetes. There was no extra education in the intervention group for better registration of the diagnoses. Pärnänen et al. [28] reported that upper respiratory infections and otitis media, hypertension, musculoskeletal pains, and diabetes were the most common reasons to visit a GP in a Finnish health center. In Denmark, the most common reasons to visit a GP were reported to be musculoskeletal, respiratory, 
TABLE 1: The distribution of the most used diagnoses in primary care doctor visits in 2006.

\begin{tabular}{|c|c|c|c|}
\hline ICD-10 & Diagnosis & Number of patients & $\%$ \\
\hline $\mathrm{J} 06.9$ & Acute upper respiratory airway infection, nonspecific & 9680 & 13,1 \\
\hline $\mathrm{I} 10$ & Primary hypertension & 2708 & 3,7 \\
\hline M54.5 & Low back pain & 2202 & 3 \\
\hline J01.0 & Acute maxillary sinusitis & 2061 & 2,8 \\
\hline E11 & Diabetes, type II & 1686 & 2,3 \\
\hline A09 & Diarrhea and gastroenteritis, presumed infectious origin & 1290 & 1,7 \\
\hline H66.9 & Acute otitis media, nonspecific & 1195 & 1,6 \\
\hline J20.9 & Acute bronchitis, nonspecific & 1184 & 1,6 \\
\hline M54 & Back pain & 988 & 1,3 \\
\hline G44.2 & Tension headache & 940 & 1,3 \\
\hline H66.0 & Acute otitis media, purulent & 854 & 1,2 \\
\hline M54.4 & Low back pain with sciatica & 647 & 0,9 \\
\hline S93.4 & Ankle sprain & 584 & 0,8 \\
\hline H10.3 & Acute conjunctivitis, nonspecific & 565 & 0,8 \\
\hline J03.9 & Acute tonsillitis, nonspecific & 540 & 0,7 \\
\hline N30.0 & Acute cystitis & 532 & 0,7 \\
\hline $\mathrm{H} 10.0$ & Conjunctivitis, purulent & 514 & 0,7 \\
\hline $\mathrm{R} 10.4$ & Abdominal pain, nonspecific & 512 & 0,7 \\
\hline H65.0 & Acute otitis media, serous & 500 & 0,7 \\
\hline J04.0 & Acute laryngitis & 487 & 0,7 \\
\hline J02.9 & Acute pharyngitis, nonspecific & 466 & 0,6 \\
\hline E78.01 & Hypercholesterolaemia, primary & 452 & 0,6 \\
\hline F43.0 & Acute stress reaction & 394 & 0,5 \\
\hline R10.3 & Lower abdominal pain & 388 & 0,5 \\
\hline M77.1 & Lateral epicondylitis & 383 & 0,5 \\
\hline $\mathrm{I} 48$ & Atrial fibrillation & 379 & 0,5 \\
\hline F32.9 & Depression, nonspecific & 370 & 0,5 \\
\hline $\mathrm{J} 45$ & Bronchial asthma & 367 & 0,5 \\
\hline I25.1 & Coronary artery disease & 352 & 0,5 \\
\hline R50.9 & Fever, nonspecific & 345 & 0,5 \\
\hline M75.1 & Rotator cuff syndrome & 338 & 0,5 \\
\hline J01.9 & Acute sinusitis, nonspecific & 319 & 0,4 \\
\hline F32 & Depression & 304 & 0,4 \\
\hline R53 & Fatigue & 291 & 0,4 \\
\hline E10 & Diabetes, type I & 289 & 0,4 \\
\hline F51.0 & Insomnia, nonorganic & 277 & 0,4 \\
\hline M53.0 & Cervicocranial syndrome & 272 & 0,4 \\
\hline R05 & Cough & 271 & 0,4 \\
\hline R51.80 & Sleep disorder, specific nonorganic & 264 & 0,4 \\
\hline
\end{tabular}

TABLE 2: Percentage of staff receiving group bonus, number of staff, and mean size of bonus in 2005 and 2006.

\begin{tabular}{|c|c|c|c|c|}
\hline \multirow{2}{*}{ Health service areas } & \multicolumn{2}{|c|}{ Percentage of staff receiving bonus } & \multicolumn{2}{|c|}{ Mean annual bonus/person $(€)$} \\
\hline & 2005 & 2006 & 2005 & 2006 \\
\hline Area 1 & 44,0 & 50,0 & 601,77 & 716,85 \\
\hline Area 2 & 16,0 & 49,0 & 205,15 & 670,97 \\
\hline Area 3 & 36,0 & 49,0 & 528,77 & 762,57 \\
\hline Area 4 & 26,0 & 41,5 & 347,85 & 592,45 \\
\hline Area 5 & 36,0 & 55,7 & 502,21 & 768,75 \\
\hline
\end{tabular}


and skin related diagnoses followed by psychological, circulatory, and metabolic disorders when the ICPC system was used [29]. Thus, the diagnoses recorded due to the present financial intervention would most likely seem to reflect real clinical life in Finnish primary care and the present intervention may provide reliable data about public health.

The main strength of the present study was the completeness of the data. The computerized patient chart system reached every single doctor in the public primary care in Espoo and Vantaa and every public primary care dentist in Espoo. The accuracy of all the diagnoses cannot be guaranteed in the present experiment. There are differences in how individual GPs code their diagnoses. However, the data were so large that differences in coding between different GPs are likely to vanish in random deviation.

\section{Limitations}

The present study does not provide clear information about whether group bonuses are applicable in other parts of healthcare. Neither does it give new answers to the key question of whether the use of these financial incentives can improve direct measures in public health or patient centered indicators of care in primary care. Qualitative studies or comparing group bonuses with other types of incentive-based interventions should be performed to gain information about how group incentives work and why there is so much variability in the effect of group bonuses.

Group bonus may alter the behavior of primary care teams. However, it is not necessarily an effective intervention for the treatment of chronic diseases and its efficacy in care teams is not very constant. The administration of healthcare must carefully consider which behavior is rewarded before intervening in clinical activity with group bonuses.

\section{Conclusion}

Group bonuses improve the completeness of diagnosis coding in primary care but the variability of this effect on the different primary care groups is substantial. They do not guarantee better recording of chronic diseases. All obtained diagnoses accurately reflected the anticipated distribution of diseases.

\section{Abbreviations}

GP: General practitioner

ITS: Interrupted time series.

\section{Conflicts of Interest}

The authors declare that they have no conflicts of interest.

\section{Acknowledgments}

The authors would like to thank the cities of Espoo and Vantaa for giving them the chance to perform this study. They would also like to thank Michael Horwood, Ph.D., for reviewing the language.

\section{References}

[1] R. L. Kane, P. E. Johnson, R. J. Town, and M. Butler, "A structured review of the effect of economic incentives on consumers' preventive behavior," American Journal of Preventive Medicine, vol. 27, no. 4, pp. 327-352, 2004.

[2] A. Giuffrida, T. Gosden, F. Forland et al., "Target payments in primary care: effects on professional practice and health care outcomes," Cochrane Database of Systematic Reviews (Online), vol. 3, article CD000531, 2000.

[3] T. Gosden, F. Forland, I. S. Kristiansen et al., "Capitation, salary, fee-for-service and mixed systems of payment: effects on the behaviour of primary care physicians," Cochrane Database of Systematic Reviews (Online), vol. 3, article CD002215, 2000.

[4] T. Doran, C. Fullwood, E. Kontopantelis, and D. Reeves, "Effect of financial incentives on inequalities in the delivery of primary clinical care in England: analysis of clinical activity indicators for the quality and outcomes framework," The Lancet, vol. 372, no. 9640, pp. 728-736, 2008.

[5] T. Doran, E. Kontopantelis, J. M. Valderas et al., "Effect of financial incentives on incentivised and non-incentivised clinical activities: longitudinal analysis of data from the UK Quality and Outcomes Framework," BMJ, vol. 342, no. jun28 1, pp. d3590d3590, 2011.

[6] E. Kontopantelis, D. Reeves, J. M. Valderas, S. Campbell, and T. Doran, "Recorded quality of primary care for patients with diabetes in England before and after the introduction of a financial incentive scheme: A longitudinal observational study," BMJ Quality \& Safety, vol. 22, no. 1, pp. 53-64, 2013.

[7] K. McLintock, A. M. Russell, S. L. Alderson et al., "The effects of financial incentives for case finding for depression in patients with diabetes and coronary heart disease: Interrupted time series analysis," BMJ Open, vol. 4, no. 8, Article ID e005178, 2014.

[8] M. J. Harrison, M. Dusheiko, M. Sutton, H. Gravelle, T. Doran, and M. Roland, "Effect of a national primary care pay for performance scheme on emergency hospital admissions for ambulatory care sensitive conditions: Controlled longitudinal study," BMJ, vol. 349, Article ID g6423, 2014.

[9] E. Kontopantelis, D. Springate, D. Reeves, D. M. Ashcroft, J. M. Valderas, and T. Doran, "Withdrawing performance indicators: Retrospective analysis of general practice performance under UK Quality and Outcomes Framework," BMJ, vol. 348, article no. g330, 2014.

[10] E. Kontopantelis, D. A. Springate, M. Ashworth, R. T. Webb, I. E. Buchan, and T. Doran, "Investigating the relationship between quality of primary care and premature mortality in England: A spatial whole-population study," BMJ, vol. 350, article no. h904, 2015.

[11] M. Weinberger, M. S. Kirkman, G. P. Saa et al., "A nurse-coordinated intervention for primary care patients with non-insulin-dependent diabetes mellitus: Impact on glycemic control and health-related quality of life," Journal of General Internal Medicine, vol. 10, no. 2, pp. 59-66, 1995.

[12] M. O. Mundinger, R. L. Kane, E. R. Lenz et al., "Primary care outcomes in patients treated by nurse practitioners or physicians: a randomized trial," The Journal of the American Medical Association, vol. 283, no. 1, pp. 59-68, 2000.

[13] S. T. Houweling, N. Kleefstra, K. J. J. Van Hateren, K. H. Groenier, B. Meyboom-de Jong, and H. J. G. Bilo, "Can diabetes management be safely transferred to practice nurses in a primary care setting? A randomised controlled trial," Journal of Clinical Nursing, vol. 20, no. 9-10, pp. 1264-1272, 2011. 
[14] K. D. Coburn, S. Marcantonio, R. Lazansky, M. Keller, and N. Davis, "Effect of a community-based nursing intervention on mortality in chronically ill older adults: A randomized controlled trial," PLoS Medicine, vol. 9, no. 7, Article ID e1001265, 2012.

[15] D. Fleming, F. Schellevis, and W. Paget, "Health monitoring in sentinel practice networks: the contribution of primary care," European Journal of Public Health, vol. 13, supplement 3, pp. 8084, 2003.

[16] P. Hjerpe, J. Merlo, H. Ohlsson, K. Bengtsson Boström, and U. Lindblad, "Validity of registration of ICD codes and prescriptions in a research database in Swedish primary care: a cross-sectional study in Skaraborg primary care database," BMC Medical Informatics and Decision Making, vol. 10, no. 1, article 23, 2010.

[17] T. Lehtovuori, T. Kauppila, J. Kallio, M. Raina, L. Suominen, and A. M. Heikkinen, "Financial team incentives improved recording of diagnoses in primary care: a quasi-experimental longitudinal follow-up study with controls," BMC Research Notes, vol. 8, no. 1, article 668, 2015.

[18] A. N. Goudswaard, K. Lam, R. P. Stolk, and G. E. H. M. Rutten, "Quality of recording of data from patients with type 2 diabetes is not a valid indicator of quality of care. A cross-sectional study," Journal of Family Practice, vol. 20, no. 2, pp. 173-177, 2003.

[19] T. Valle, V. A. Koivisto, A. Reunanen, T. Kangas, and A. Rissanen, "Glycemic control in patients with diabetes in Finland," Diabetes Care, vol. 22, no. 4, pp. 575-579, 1999.

[20] E. Ketola, R. Sipilä, M. Mäkelä, and M. Klockars, "Quality improvement programme for cardiovascular disease risk factor recording in primary care," Quality \& Safety in Health Care, vol. 9, no. 3, pp. 175-180, 2000.

[21] Effective Practice and Organisation of Care (EPOC), "Interrupted time series (ITS) analyses," EPOC Resources for review authors. Oslo: Norwegian Knowledge Centre for the Health Services; http://epoc.cochrane.org/epoc-specific-resources-reviewauthors, 2013.

[22] M. B. Rosenthal, R. G. Frank, Z. Li, and A. M. Epstein, "Early experience with pay-for-performance: From concept to practice," Journal of the American Medical Association, vol. 294, no. 14, pp. 1788-1793, 2005.

[23] K. Kirschner, J. Braspenning, J. E. A. Jacobs, and R. Grol, “Design choices made by target users for a pay-for-performance program in primary care: An action research approach," $B M C$ Family Practice, vol. 13, article no. 25, pp. 161-171, 2012.

[24] S. M. Campbell, D. Reeves, E. Kontopantelis, B. Sibbald, and M. Roland, "Effects of pay for performance on the quality of primary care in England," The New England Journal of Medicine, vol. 361, no. 4, pp. 368-378, 2009.

[25] A. Scott, P. Sivey, D. Ait Ouakrim et al., "The effect of financial incentives on the quality of health care provided by primary care physicians," Cochrane Database of Systematic Reviews, no. 9, Article ID CD008451, 2011.

[26] B. Yuan, L. He, Q. Meng, and L. Jia, "Payment methods for outpatient care facilities," Cochrane Database of Systematic Reviews, vol. 2017, no. 3, Article ID CD011153, 2017.

[27] D. B. Balkin and E. F. Montemayor, "Explaining Team-Based Pay: A Contingency Perspective Based on the Organizational Life Cycle, Team Design, and Organizational Learning Literatures," Human Resource Management Review, vol. 10, no. 3, pp. 249-269, 2000

[28] H. Pärnänen, E. Kumpusalo, and J. Takala, "Primary health care ICD - A tool for general practice research," International Journal of Health Planning and Management, vol. 15, no. 2, pp. 133-148, 2000

[29] G. Moth, F. Olesen, and P. Vedsted, "Reasons for encounter and disease patterns in Danish primary care: changes over 16 years," Scandinavian Journal of Primary Health Care, vol. 30, no. 2, pp. 70-75, 2012. 


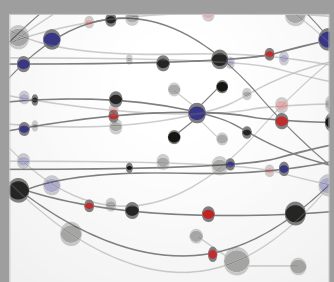

The Scientific World Journal
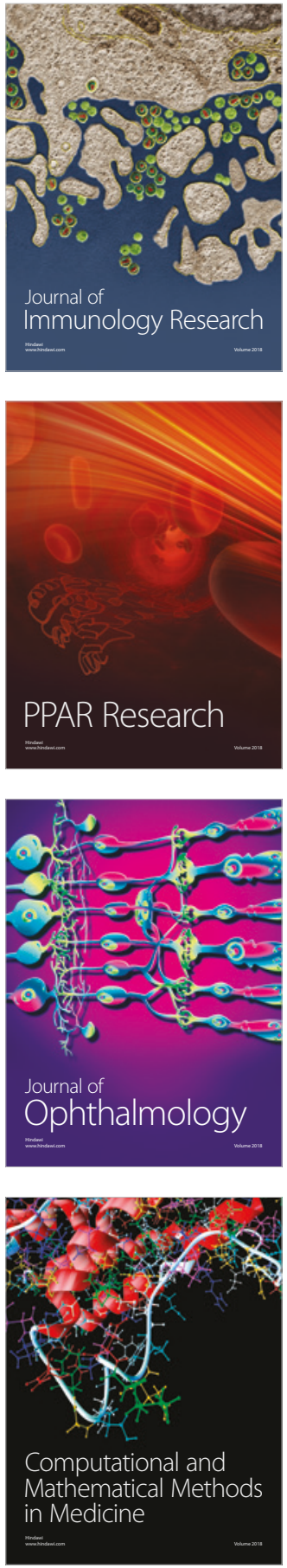

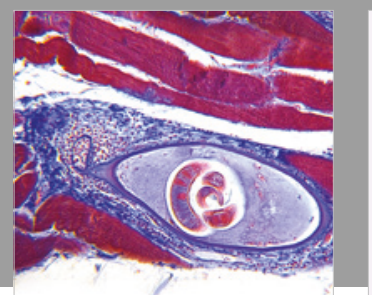

Gastroenterology Research and Practice

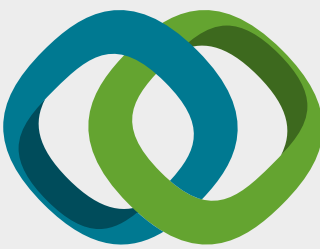

\section{Hindawi}

Submit your manuscripts at

www.hindawi.com


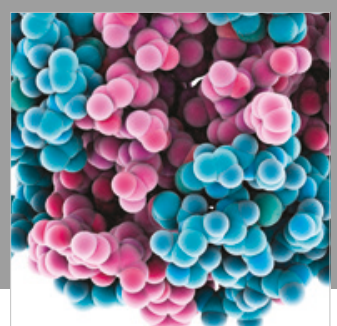

ournal of

Diabetes Research

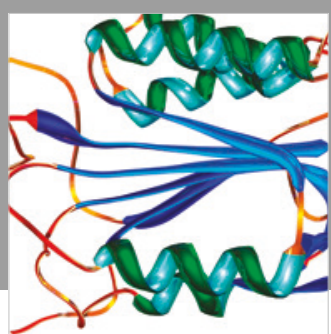

Disease Markers
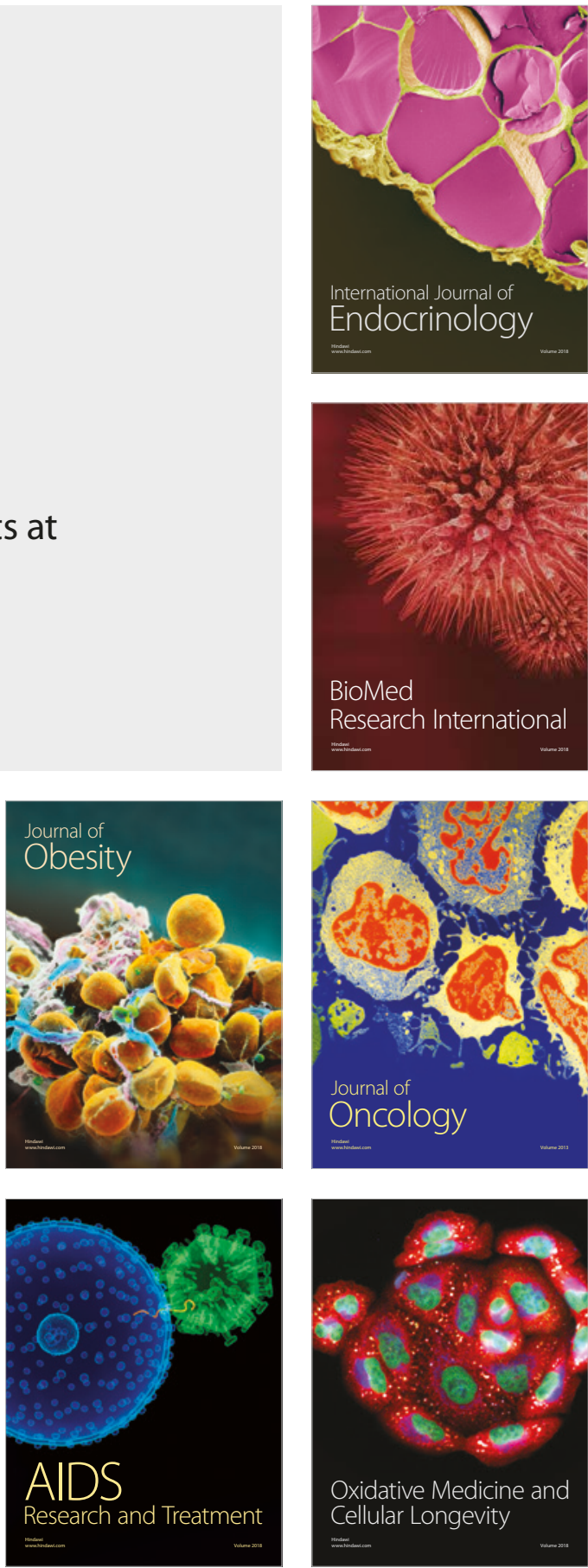